\title{
Methods and Applications of Power Series
}

\author{
By Jay A. Leavitt
}

Power series in the past played a minor role in the numerical solutions of ordinary and partial differential equations. There have been good reasons. It is often difficult to operate with power series. Finding the series expansion of

$$
\frac{d^{k} u}{d x^{k}}=F\left(x, \frac{d u}{d x}, \cdots, \frac{d^{k-1} u}{d x^{k-1}}\right)
$$

can be arduous. Furthermore if the series can be found, often it will converge in too small a region. There are, however, certain advantages which make their use desirable. A truncated series forms a closed approximation of the solution which can be evaluated at any point in the region where the series converges. Instability, which causes difficulties for finite difference solutions, does not affect the power series solutions. The series solution, with its great accuracy, permits study of the analytic properties of the solution to an extent which is unachievable with a finite difference solution; and the series solution can be used as an intermediate result which can be integrated and differentiated easily. If a finite difference solution is only an intermediate step in the solution of a problem, computer storage problems can be a major concern. Derivatives and interpolated values of the difference solution can be very unreliable. In this paper we intend to show how many of the disadvantages of power series can be overcome by automatic coding procedures and to indicate some of their useful properties and results.

In this paper we use the convention that a sum is zero if the upper limit is less than the lower.

Let $P(I)$ denote the coefficient of $x^{I-1}$ in the polynomial $P(x)=\sum_{I=1}^{N} P(I) x^{I-1}$ and let $Q(I, J)$ denote the coefficient of $x^{I-1} y^{J-1}$ in the polynomial $Q(x, y)=$ $\sum_{I=1}^{N} \sum_{J=1}^{N} Q(I, J) x^{I-1} y^{J-1}$.

The following formulas for integration and differentiation are well known:

If $Q(x, y)=\int^{x} P(x, y) d x$ or $R(x, y)=\int^{y} P(x, y) d y$ then

$$
\begin{gathered}
Q(I, J)=P(I-1, J) /(I-1) \quad \text { and } R(I, J)=P(I, J-1) /(J-1) . \\
\text { If } Q(x, y)=\partial P(x, y) / \partial x \text { or } R(x, y)=\partial P(x, y) / \partial y \text { then } \\
\qquad Q(I, J)=I \cdot P(I+1, J) \text { and } R(I, J)=J \cdot P(I, J+1) .
\end{gathered}
$$

The formulas for multiplication and division are as follows:

If $R(x, y)=P(x, y) \cdot Q(x, y)$ then

$$
R(I, J)=\sum_{L=1}^{I} \sum_{M=1}^{J} P(L, M) Q(I-L+1, J-M+1) .
$$

If $R(x, y)=P(x, y) / Q(x, y)$ then

$$
\begin{aligned}
& R(I, J)=\frac{1}{Q(1,1)}\left(P(I, J)-\sum_{K=1}^{I-1} \sum_{L=1}^{J} R(K, L) Q(I-K+1, J-L+1)\right. \\
&\left.-\sum_{L=1}^{J-1} R(I, L) Q(1, J-L+1)\right) .
\end{aligned}
$$

They have been used in machine calculations by R. D. Richtmyer [1].

Received March 22, 1965. 
The formula for inverting a power series is available in many calculus texts:

If $Q=Q(1)+\sum_{K=2}^{\infty} Q(K)(P-P(1))^{K-1}$ and $P=P(1)+\sum_{J=2}^{\infty} P(J)$. $(Q-Q(1))^{J-1}$ then

$$
P(2)=1 / Q(2) \text { and } P(K)=-\sum_{I=2}^{K-1} P(I)(Q-Q(1))_{K}^{I-1} / Q(2)^{K-1}
$$

where $(Q-Q(1))_{K}^{I-1}$ is the coefficient of $(P-P(1))^{K-1}$ in the expansion of $(Q-Q(1))^{I}(K=3,4, \cdots)$. The expansion of $(Q-Q(1))^{I}$ can be found by using the multiplication algorithm or by the formula for finding a power of a polynomial (see below).

The next set of formulas is for determining the expansions of functions of a function in two variables, $R(x, y)=P(Q(x, y))$. If $A$ is the region of radius $r$ where $P$ converges, then the expansion $R$ is a valid representation of $P(Q)$ in the region where $Q$ converges and $|Q| \leqq r$. The algorithms, $\mathrm{A}, \mathrm{B}, \mathrm{C}$, and $\mathrm{D}$, below, were derived by using Leibnitz' rule of differentiation.

A. If

(1) $Q(x, y)=P(x, y)^{z}$ where $z$ is any positive or negative real number then

(2) $\partial Q / \partial y=z Q(\partial P / \partial y) / P$ or

(3) $P \partial Q / \partial y=z Q \partial P / \partial y$.

Applying the differential operators $D_{x}$ and $D_{y}$ which stand for $\partial / \partial x$ and $\partial / \partial y$ respectively, we find:

(4) $D_{y}{ }^{J-1}(P \partial Q / \partial y)=D_{v}{ }^{J-1}(z Q \partial P / \partial y)$ and

(5) $D_{x}{ }^{I-1} D_{y}{ }^{J-1}(P \partial Q / \partial x)=D_{x}{ }^{I-1} D_{y}{ }^{J-1}(z Q \partial P / \partial x)$.

We apply Leibnitz' rule of differentiation to (4) and (5) and express the derivatives in terms of the coefficients:

$$
\begin{aligned}
Q(1,1) & =P(1,1)^{z}, \\
Q(1, J+1) & =\left(\sum_{L=1}^{J} \frac{L(z+1)-J}{J}\right.
\end{aligned}
$$

$$
\begin{array}{r}
\cdot Q(1, J+1-L) P(1, L+1)) / P(1,1) \\
Q(I+1, J)=\begin{array}{rr}
(\operatorname{sum} 1+\operatorname{sum} 2) / P(1,1) & (J=1,2, \cdots, N-1), \\
\text { sum } 1= & z P(I+1,1) Q(1, J) \\
& +\sum_{L=1}^{J-1}(z P(I+1, L+1) Q(1, J-L)
\end{array} \\
\operatorname{sum} 2=\sum_{L=1}^{I-1} \sum_{K=0}^{J-1} \frac{L(z+1)-I}{I} Q(I-L+1, J-K) P(L+1, K+1) .
\end{array}
$$

The formulas $\left(1^{\prime}\right)$ and $\left(2^{\prime}\right)$ serve as the algorithms for finding the coefficients of the power of a series in one variable.

B. If

(1) $Q(x, y)=\exp (P(x, y))$ then

(2) $\partial Q / \partial y=Q \partial P / \partial y$ and 
(3) $D_{y}{ }^{J-1} \partial Q / \partial y=D_{y}{ }^{J-1}(Q \partial P / \partial y), J=1, \cdots, N-1$,

(4) $D_{x}{ }^{I-1} D_{y}{ }^{J-1} \partial Q / \partial x=D_{x}{ }^{I-1} D_{y}{ }^{J-1}(Q \partial P / \partial x), I=1, \cdots, N-1 ; J=1, \cdots, N$ In terms of the coefficients these equations become:

$\left(1^{\prime}\right) Q(1,1)=\exp (P(1,1))$

(2') $Q(1, J+1)=(1 / J) \sum_{L=0}^{J-1}(L+1) P(1, L+2) Q(1, J-L), J=$ $1, \cdots, N-1$,

C. If

$$
\begin{aligned}
& Q(I+1, J)=(1 / I) \sum_{K=0}^{I-1} \sum_{L=0}^{J-1}(K+1) P(K+2, L+1) \\
& \cdot Q(I-K, J-L), \quad I=1, \cdots, N-1 ; J=1, \cdots, N .
\end{aligned}
$$

(1) $Q(x, y)=\log (P(x, y))$ then

(2) $\partial Q / \partial y=(\partial P / \partial y) / P$ and

(3) $D_{y}{ }^{J-1}(P \partial Q / \partial y)=D_{y}{ }^{J-1} \partial P / \partial y$,

(4) $D_{x}{ }^{I-1} D_{y}{ }^{J-1}(P \partial Q / \partial x)=D_{x}{ }^{l-1} D_{y}{ }^{J-1} \partial P / \partial x$.

Again, using Liebnitz' rule of differentiation and rearranging terms, we find that the formulas for the coefficients are:

$\left(1^{\prime}\right) Q(1,1)=\log (P(1,1))$,

$\left(2^{\prime}\right) Q(1, J+1)=\left(P(1, J+1)-(1 / J) \sum_{K=1}^{J-1}(J-K) Q(1, J-K+1)\right.$. $P(1, K+1)) / P(1,1), J=1, \cdots, N-1$,

$$
Q(I+1, J)=\left(P(I+1, J)-\sum_{L=1}^{J-1} P(1, L+1) Q(I+1, J-L)\right.
$$

$$
\begin{array}{r}
\left.-(1 / I) \sum_{K=1}^{I-1} \sum_{L=0}^{J-1}(I-K) P(K+1, L+1) Q(I-K+1, J-L)\right) / P(1,1) \\
I=1, \cdots, N-1 ; J=1, \cdots, N .
\end{array}
$$

D. The sine and cosine of a series are found by: If

(1) $Q(x, y)=\sin (P(x, y)), R(x, y)=\cos (P(x, y))$ then

(2) $\partial Q / \partial y=R \partial P / \partial y, \partial R / \partial y=-Q \partial P / \partial y$ and

(3) $D_{y}{ }^{I-1} \partial Q / \partial y=D_{y}{ }^{I-1}(R \partial P / \partial y), D_{y}{ }^{l-1} \partial R / \partial y=D_{y}{ }^{I-1}(-Q \partial P / \partial y)$,

(4) $D_{x}{ }^{J-1} D_{y}{ }^{I-1} \partial Q / \partial x=D_{x}{ }^{J-1} D_{y}{ }^{I-1}(R \partial P / \partial x), D_{x}{ }^{J-1} D_{y}{ }^{I-1} \partial R / \partial x=D_{x}{ }^{J-1} D_{y}{ }^{I-1}$ $(-Q \partial P / \partial x)$

or, in terms of the coefficients we find:

$\left(1^{\prime}\right) Q(1,1)=\sin (P(1,1)), R(1,1)=\cos (P(1,1))$,

$$
Q(1, I+1)=(1 / I) \sum_{K=0}^{I-1}(I-K) R(1, K+1) P(1, I-K+1),
$$

$\left(2^{\prime}\right) \quad R(1, I+1)=-(1 / I) \sum_{K=0}^{I-1}(I-K) Q(1, K+1) P(1, I-K+1)$,

$$
\begin{aligned}
& \quad I=1, \cdots, N-1, \\
& Q(J+1, I)=(1 / J) \sum_{L=0}^{J-1} \sum_{K=0}^{I-1}(J-L) P(J-L+1, I-L) \\
& \cdot R(L+1, K+1), \\
& R(J+1, I)=-(1 / J) \sum_{L=0}^{J-1} \sum_{K=0}^{I-1}(J-L) P(J-L+1, I-L) \\
& \cdot Q(L+1, K+1), \quad J=1, \cdots, N-1 ; I=1, \cdots, N .
\end{aligned}
$$


The following formulas for a function of a function depend upon the above methods for the evaluation of their coefficients. These algorithms are for series in one variable.

E. The representation of the Bessel Function of order zero can be calculated directly, even though the argument is a polynomial:

$$
Q(x)=J_{0}(P(x)) \cong \sum_{K=0}^{N}\left(-(P / 2)^{2}\right)^{K} /(K !)^{2} .
$$

Nesting is used to form the sum. The indicated square is formed using the algorithms A. $P$ is of degree $N-1$. It should be noted that the coefficients $Q(I)$ are obtained exactly only if $P(1)=0$.

$F$. The arctangent, arcsine, and arccosine of a series are found directly from their classical definitions:

$$
\begin{array}{ll}
Q(x)=\tan ^{-1}(P,(x))=\int \frac{d P / d x}{1+P^{2}} d x, & Q(1)=\tan ^{-1}(P(1)), \\
Q(x)=\sin ^{-1}(P(x))=\int \frac{d P / d x}{\sqrt{ }\left(1-P^{2}\right)} d x, & Q(1)=\sin ^{-1}(P(1)), \\
Q(x)=\cos ^{-1}(P(x))=\int \frac{d P / d x}{\sqrt{ }\left(1-P^{2}\right)} d x, & Q(1)=\cos ^{-1}(P(1)) .
\end{array}
$$

These expressions are found by using the power, division or multiplication, differentiation and integration formulas.

G. The formulas for the Chebyshev, Legendre, Hermite and Laguerre polynomials as functions of a function are derived from their generating functions (see Courant and Hilbert [2]):

If $Q(x)=T_{N}(P(x))$ where $T_{N}$ is the Chebyshev polynomial of degree $N$, then $T_{N}(P)$ is the coefficient of $y^{N}$ in the expansion of $\left(1-.25 y^{2}\right) /\left(1-P y+.25 y^{2}\right)$, $P$ is a polynomial in one variable. The division algorithm handles this expression easily.

If $Q(x)=L_{N}(P(x))$ where $L_{N}$ is the Legendre polynomial of degree $N$, then $L_{N}(P)$ is the coefficient of $y^{N}$ in the expansion of $\left(1-2 P y+y^{2}\right)^{-1 / 2}$. The formulas A serve to carry out the indicated square root.

If $Q(x)=H_{N}(P(x))$ where $H_{N}$ is the Hermite polynomial of degree $N$, then $H_{N}(P)$ is the coefficient of $y^{N} / N$ ! in $\exp \left(-y^{2}+2 P y\right)$.

Similarly the Laguerre polynomial $\operatorname{La}_{N}(P)$ is found from the expansion of $\exp (-P y /(1-y)) /(1-y)$; it is the coefficient of $y^{N} / N$ !.

The Jacobi polynomial can be handled in the same manner.

The above formulas indicate the versatility of the methods. Not only is it possible to perform algebraic operations on polynomials, but also the series representation of many of the elementary functions can easily be found. If the above formulas are used to form $R=P(Q)$ when $Q$ is just the variable $x$, then the classical expressions result. The formula for the Chebyshev polynomials of $x$ yielded $T_{0}, T_{1}, \cdots, T_{25}$ in about 1 minute on the CDC 1604. These routines, in general, were found to be very fast in dealing with compound functions of one variable.

Power series methods are well suited for initial value problems of ordinary and partial differential equations. The Cauchy-Kowalewsky theorem provides the existence of and method of finding the solution; it is a power series solution. 
These methods are also useful for solving second order nonlinear boundary value problems of ordinary differential equations. An often used approach to the nonlinear boundary value problem is to solve an initial value problem which satisfies the same differential equation. The initial value of the first derivative of the solution to the initial value problem is varied until the solution passes through the prescribed endpoint of the boundary value problem. That is, if we want to solve: $d^{2} P / d x^{2}=f\left(x, P, P^{\prime}\right), P\left(x_{0}\right)=P_{0}, P\left(x_{1}\right)=P_{1}, P^{\prime}=d P / d x$, then we would guess $P^{\prime}\left(x_{0}\right)$ and solve the initial value problem: $d^{2} P^{i} / d x^{2}=f\left(x, P^{i}, P^{i^{\prime}}\right), P^{i}\left(x_{0}\right)=$ $P_{0}, P^{i^{\prime}}\left(x_{0}\right) \doteq y^{i}$. $P^{i}$ represents the solution corresponding to the $i$ th guess, $y^{i}$, of the initial data $P^{\prime}\left(x_{0}\right)$. The successive guesses $y^{i}$ are chosen by interpolation so that Limit $_{i \rightarrow \infty} P^{i}\left(x_{1}\right)=P_{1}$. The disadvantage of this technique is that the solution must be recalculated after each interpolation and the sequence $P^{i}\left(x_{1}\right)$ may converge slowly, if at all, to $P_{1}$.

The following alternative solution is proposed. Consider the following system:

$$
\begin{array}{ll}
\partial P / \partial x=Q(x, y), & P\left(x_{0}, y\right)=P_{0}, \\
\partial Q / \partial x=f(x, P(x, y), Q(x, y)), & Q\left(x_{0}, y\right)=y .
\end{array}
$$

The truncated solution $P(x, y)=\sum_{i=0}^{N} \sum_{j=0}^{N} p_{i j} x^{i} y^{j}$ can be evaluated at $x_{1}$ which gives a polynomial in $y$ :

$$
P\left(x_{1}, y\right)=\sum_{i=0}^{N} \sum_{j=0}^{N} p_{i j} x_{1}^{i} y^{j}=P_{1} .
$$

The zeroes, $y_{k}$, of this equation provide the appropriate initial values of the derivative $P^{\prime}\left(x_{0}\right)=y_{k}$ such that $\sum_{i=0}^{N} \sum_{j=0}^{N} p_{i j} x_{1}{ }^{i} y^{j}{ }_{k}=P_{1}$ and $P\left(x, y_{k}\right)$ satisfies the original boundary value problem.

An important drawback is that series solutions to differential equations may converge in too small a region. M. D. Van Dyke [3] discusses why some attempts with power series solutions of supersonic flows involving shock waves have failed.

The first complete solution of one of these problems was presented by G. Lewis [4]. He solved the problem of supersonic flow past a blunt body with axial symmetry. The shape of the shock wave is assumed and taken as the initial line. The conditions ahead of the shock are also assumed and the Rankine-Hugoniot conditions provide the initial conditions. Thus the problem is expressed as a Cauchy problem. However, as M. D. Van Dyke points out, the solution does not converge up to the body because of a line of singularities, upstream from the shock, which is closer to the initial line than is the body. The problem is expressed as 4 nonlinear partial differential equations in 2 variables. ${ }^{1}$

G. Lewis has developed and proved the convergence of two methods of analytic continuation which he used to extend the solution up to the body. One method is simply a reexpansion of the series solution about another point with some extra truncation of the new series. The other method of continuation, which is more accurate, takes advantage of the flow equations. The initial series are calculated at, say, $p_{0}$ on the shock and are found to converge in a region including the point $p_{1}$. From the differential equations new series are computed about $p_{1}$, using the values

\footnotetext{
${ }^{1}$ Another solution of this problem was given earlier without proof by A. Van Tuyl [6].
} 
at $p_{1}$ of the original series for the initial data. G. Lewis calculated the solution on an IBM 704 which is an 8-place machine. He used unnormalized double precision arithmetic and obtained a solution with 8-place accuracy.

The author and Lewis, independently, have recalculated the solution using a bilinear mapping to map the line of singularities further away from the body. This allowed the body to be reached without the need of analytic continuation. The author's calculations were carried out in single precision (10 places) on a CDC 1604 . The machine has built-in rounding operations. The location of the body agreed to 7 places with the original calculations of G. Lewis, obtained by analytic continuation. Consistency checks for the solution obtained by mapping indicated full 10place accuracy all the way up to the body. The bilinear mapping, used by the author, sent the origin (on the shock) into the origin and a point near the body into itself. The real line was mapped onto itself, and a parameter was used to maximize the accuracy. Accuracy was determined by the 2 consistency checks of the problem:

Bernoulli's Law: $2 \gamma p /(\gamma-1) \rho+u^{2}+v^{2}=$ constant and

the gas law: $\quad p=A(S) \rho^{\gamma}$,

$A(S)$ is constant along a streamline, $\gamma$ is the gas constant, and $S$ is the entropy. These equations were available as consistency checks since they were not used in the calculation of the flow.

The calculation of the series through degree 24 took 100-135 seconds.

These methods were. also used by the author [5] to calculate supersonic flows past conical shocks. For this initial value problem there are 5 nonlinear partial differential equations in 2 variables. The calculation was performed on an IBM 7090 using 8-place single precision significance arithmetic. 7- to 8-place accuracy was obtained for the flow where the shock was taken as a right circular cone. The general flow, where the cross-section of the shock was taken to be an ellipse, involves a singularity. Outside a neighborhood of the singularity 5- to 7-place accuracy was achieved. The expansions for the flow variables, pressure, density, and velocity were calculated on a constant spherical surface between the shock and the body along several radial lines running from the shock to the center of the ellipse. The maximum number of analytic continuations necessary to reach the body along any radial line was three. Each continuation resulted in the loss of 1 decimal place of accuracy. By calculating up to the singularity from different directions it was found that the singularity behaves like a branch point. Calculations from separate directions gave different results in a neighborhood of the singularity, although they agreed in overlapping regions away from the singularity.

From several points of view power series have shown themselves useful. They are not only a computational tool with a high degree of accuracy, but they also provide an efficient method for generating polynomial expressions which are important in themselves, for example the Chebyshev polynomials. By mapping or analytic continuation, power series solutions may be extended into the large. Stability criteria do not affect power series solutions. The techniques are valid even when the equations change type, i.e., from elliptic to hyperbolic as in the case of flow past a 
blunt body. A power series form of solution is much more compact than a finite difference solution, and it allows study of the analytic behavior of the solution.

University of Minnesota

Minneapolis, Minnesota

1. R. D. Richtmyer, Detached-Shock Calculations by Power Series, I, A.E.C. Research and Development Report, NYU-7973, New York University, Courant Inst. of Math. Sci., 1957.

2. R. Courant \& D. Hilbert, Methoden der mathematischen Physik, Vols. I (2nd ed.), II, Springer, Berlin, 1931, 1937; English transl., Vols. I (2nd ed.), II, Interscience, New York, 1953, 1962. MR 6, 97; MR 13, 800; MR 16, 426; MR $25 * 4216$.

3. M. D. VAN DYKe, "A model of supersonic flow past blunt axi-symmetric bodies, with application to Chester's solution," J. Fluid Mech., v. 3, 1958, pp. 515-522. MR 19, 915.

4. G. Lwwis, Two Methods Using Power Series for Solving Analytic Initial Value Problems, A.E.C. Research and Development Report, NYU-2881, New York University, Courant Inst. of Math. Sci., 1960.

5. J. A. Lanvirt, A Power Series Solution for Compressible Flow Past a Conical Shock Wave, A.E.C. Research and Development Report, NYU-10, 432, New York University, Courant Inst. of Math. Sci., 1963.

6. A. VAN TUYL, The Use of Rational Approximations in the Calculation of Flows with Detached Shocks, NAVORD Report No. 6697, Naval Ordnance Lab., Silver Spring, Md., Nov., 1959. 